\title{
Experiencias sexuales y malestar asociado en personas jóvenes homosexuales
}

\author{
Juan Enrique Nebot-Garcia \\ junebot@uji.es \\ Vicente Morell-Mengual \\ vicente.morell@uv.es \\ Estefanía Ruiz-Palomino \\ eruiz@uji.es \\ Cristina Giménez-García \\ gimenezc@uji.es
}

\section{Resumen}

Introducción: Las etiquetas sobre orientación sexual suelen ser muy rígidas, creando malestar en las personas que tienen un comportamiento sexual diferente al heteronormativo. En este contexto, la edad podría tener un factor modulador en el malestar experimentado.

Método: En el estudio participaron un total de 90 personas homosexuales, repartidas equitativamente en tres grupos de edad: de 18 a 22 años, de 23 a 27 años y de 28 a 33 años. La media de edad fue de 24,81 (DT =4,11), siendo en cada grupo el $50 \%$ hombres y el $50 \%$ mujeres. Además de los datos sociodemográficos, todos contestaron un cuestionario sobre la vivencia de su orientación sexual.

Resultados: Entre los grupos de edad, no se obtuvieron diferencias significativas en el porcentaje de personas que habían tenido fantasías, deseos y conductas sexuales, tanto con el mismo sexo como con el opuesto. No obstante, a nivel descriptivo, se observó una tendencia del grupo más joven a sentir más malestar que el resto de grupos, sobre todo, en las experiencias con el mismo sexo.

Conclusiones: Existe un patrón diferencial de malestar que es más evidente entre la población joven, sobre todo, en cuanto a las conductas homosexuales. Estas diferencias de edad podrían estar relacionadas con la madurez de los participantes, siendo los más jóvenes a quienes les afecte más los posibles prejuicios de la sociedad. Habría que investigar detalladamente estos resultados para conocer más sobre la problemática de estos jóvenes, con el fin de elaborar programas de atención específicos para esta población.

Palabras clave: homosexualidad, orientación sexual, edad, experiencias sexuales, malestar. 


\begin{abstract}
Introduction: The labels on sexual orientation are usually very rigid, creating discomfort in people who have a sexual behavior different from heteronormativity. In this context, age may moderate the discomfort experience.

Method: A total of 90 homosexual people participated in the study, divided equally into three age groups: from 18 to 22 years old, from 23 to 27 years old and from 28 to 33 years old. The mean age was 24.81 (SD = 4.11), being $50 \%$ men and $50 \%$ women in each group. In addition to the sociodemographic data, all the participants answered a questionnaire about the experience of their sexual orientation.

Results: Among the age groups, no statistical significant differences were found in the percentage of people who had fantasies, desires and sexual behaviors, both with the same sex and with the opposite sex. However, at a descriptive level, there was a tendency for the younger group to feel more discomfort than the rest of the groups, especially in the experiences with the same sex.

Conclusions: There is a differential pattern of discomfort that is more evident among the young population, especially in terms of homosexual behavior. These age differences could be related to the maturity of the participants, being the youngest those who are more affected by the possible prejudices of society. It would be necessary to investigate these results in detail to learn more about the problems of these young people, in order to develop specific care programs for this population.
\end{abstract}

Keywords: homosexuality, sexual orientation, age, sexual experiences, discomfort.

\title{
Introducción
}

Durante la definición de la propia orientación sexual, las personas pasan por una serie de fases o etapas hasta llegar a la aceptación e integración de su orientación sexual dentro de su identidad como persona. Este camino puede llegar a ser largo y complicado, sobre todo, para aquellas personas que se diferencian de la norma social, como es el caso de las personas homosexuales (Soriano 2004).

No obstante, no solo la población homosexual sufre esta presión heteronormativa, sino que el propio colectivo heterosexual se ve condicionado al tener que ajustarse a unos comportamientos esperados, provocando malestar en aquellas personas que se alejan de la norma (Bosson, Prewitt-Freilino y Taylor 2005). Y es que las etiquetas sexuales son, en muchos casos, demasiado rígidas (Epstein, McKinney, Fox y Garcia 2012; Thompson y Morgan 2008), encorsetando a las personas que se sitúan en ellas. De hecho, existe un número elevado de población heterosexual que se ha cuestionado su orientación sexual (Morgan, Steiner y Thompson 2010; Morgan y Thompson 2011) o ha tenido algún comportamiento homosexual a lo largo de su vida (Copen, Chandra y Febo-Vazquez 2016; Nebot-Garcia, García-Barba, Gil-Juliá, Giménez-García y Ballester-Arnal 2018).

En la actualidad, continúa vigente la heteronormatividad, una creencia que considera que la heterosexualidad es la única orientación válida, normal y natural, dando por sentado que todas las personas son heterosexuales si no indican lo contrario (Herz y Johansson 2015). Esta creencia tiene diversos efectos en la sociedad: por una parte, la discriminación que sufre la población homosexual por no ajustarse a la norma 
(Digoix et al. 2016; Martxueta y Etxeberria 2014) y, por otra parte, la homofobia interiorizada, es decir, los prejuicios que integran algunas personas homosexuales sobre ellas mismas (Newcomb y Mustanski 2010). Es por ello que el hecho de ser homosexual o tener conductas homosexuales se pueda vivir con un elevado sentimiento de malestar.

El proceso identitario por el que pasan las personas homosexuales puede generarles un sentimiento de diferencia que puede provocarles una serie de dudas sobre quiénes son realmente. Esta confusión, ligada a la presión por seguir la norma social, puede llevar a las personas homosexuales a tener relaciones sexuales con gente del otro sexo, tanto para probar como para reafirmar que no son homosexuales. No obstante, estos intentos por amoldarse a lo esperado y por ocultar la verdadera orientación sexual pueden llegar a ser muy disonantes y contraproducentes para la propia persona (Soriano 2004).

Uno de los factores que puede influir en el proceso identitario es la edad de los participantes (Luyckx, Klimstra, Duriez, Van Petegem y Beyers 2013), ya que la etapa madurativa en la que se encuentren va a ser determinante en lo consolidada que esté su identidad y en el grado de afectación que experimenten por la presión social, siendo los jóvenes los que más malestar sufran (Kertzner, Meyer, Frost y Stirratt 2009).

Por ello, el objetivo del presente trabajo es analizar si existen diferencias en las experiencias sexuales y el malestar asociado en función de la edad de la población joven que se identifica como homosexual. Teniendo en cuenta este objetivo, se plantean las siguientes hipótesis: 1) mayor porcentaje de población joven habrá experimentado malestar al haber realizado conductas homosexuales; 2) mayor porcentaje de población joven habrá experimentado malestar al haber realizado conductas heterosexuales.

\section{Método}

\section{Participantes}

Del total de la muestra, se seleccionaron 90 personas homosexuales, siendo equitativo el número en los tres grupos de edad: de 18 a 22 años, de 23 a 27 años y de 28 a 33 años. La media de edad fue de 24,81 años (DT $=4,11$ ), siendo en cada grupo el $50 \%$ hombres y el $50 \%$ mujeres. La mayoría de participantes tenían estudios universitarios, eran ateos o agnósticos y progresistas (véase la tabla 1). Para poder ser incluidos en el estudio, los participantes debían tener entre 18 y 33 años y autoidentificarse como homosexuales.

Tabla 1. Datos sociodemográficos de los participantes.

\begin{tabular}{llc}
\hline Variables sociodemográficas & $\%$ \\
\hline Creencias religiosas & Creyente practicante & 4,4 \\
\cline { 2 - 3 } & Creyente no practicante & 14,4 \\
\cline { 2 - 3 } & Ateo o agnóstico & 81,2 \\
\hline \multirow{2}{*}{ Nivel de estudios } & Sin estudios & 1,1 \\
\cline { 2 - 3 } & Estudios primarios & 1,1 \\
\cline { 2 - 3 } & Estudios secundarios & 12,2 \\
\cline { 2 - 2 } &
\end{tabular}




\begin{tabular}{llc}
\cline { 2 - 2 } & \multicolumn{1}{l}{ Formación profesional } & 21,1 \\
\cline { 2 - 3 } & Diplomatura & 3,3 \\
\cline { 2 - 3 } & Licenciatura/grado & 42,2 \\
\cline { 2 - 3 } & Máster o Doctorado & 19 \\
\hline Ideología política & Conservador & 2,2 \\
\cline { 2 - 3 } & Progresista & 63,3 \\
\cline { 2 - 3 } & Centro & 12,3 \\
\cline { 2 - 3 } & Indiferente & 22,2 \\
\hline
\end{tabular}

\section{Instrumentos}

Para este estudio se utilizó un cuestionario ad hoc, en el que se evaluaba la vivencia sobre la propia sexualidad y la orientación sexual. Además de algunas preguntas sociodemográficas, este instrumento consta de dos escalas con el mismo formato, una para valorar la experiencia homosexual y otra, la heterosexual. Ambas constan de 6 ítems que evalúan si alguna vez han considerado que alguna persona del mismo sexo o del otro sexo era guapa (ítem 1) o atractiva (ítem 2), si han tenido sueños eróticos (ítem 3), fantasías (ítem 4) o deseo de tener relaciones sexuales (incluyendo tocamientos o masturbación) con alguien del mismo sexo o del otro sexo (ítem 5), o si, finalmente, han tenido relaciones sexuales (incluyendo tocamientos o masturbación) con alguien del mismo sexo o del otro sexo (ítem 6). Las preguntas deben contestarse diciendo sí o no. Si en alguno de los ítems se responde afirmativamente, se debe contestar si el hecho de realizar esa conducta le ha generado malestar o no. Para más información sobre el cuestionario, consulten a los autores.

\section{Procedimiento}

Para conseguir la muestra de participantes, se hizo difusión de la investigación por redes sociales y foros de interés. Una vez accedían al formulario online desde sus casas, en la primera pantalla se les explicaba que la participación era voluntaria y que sus respuestas eran anónimas y confidenciales. Además, antes de cumplimentar el cuestionario, se les pedía el consentimiento informado.

\section{Análisis estadísticos}

Mediante el programa estadístico SPSS versión 24 se realizaron, en primera instancia, análisis de frecuencias y descriptivos para obtener los datos demográficos de las personas participantes. Asimismo, para analizar las diferencias de los grupos de edad en la vivencia de experiencias homosexuales y heterosexuales y el malestar asociado, se realizaron tablas de contingencias y pruebas de chi cuadrado.

\section{Resultados}

Entre los grupos de edad, no se obtuvieron diferencias significativas en el porcentaje de personas que habían tenido experiencias homosexuales ni heterosexuales, así como tampoco hubo diferencias significativas en el malestar experimentado. 
No obstante, a nivel descriptivo, en el grupo de menor edad más personas mostraron malestar en las experiencias homosexuales, en comparación con el resto de grupos. Por lo que respecta a las conductas en concreto, a nivel general, se observa una elevada prevalencia de ocurrencia en todas ellas siendo de un $90 \%$ o superior. En concreto, la más frecuente para los tres grupos fue el hecho de haber considerado a una persona del mismo sexo como guapa; del mismo modo, también fue la que mayor malestar registró. Por el contrario, la que menos prevalencia muestra es el hecho de haber tenido relaciones sexuales con alguien del mismo sexo que, a su vez, también es la variable que menor malestar registra (véase la tabla 2).

Tabla 2. Porcentaje de personas de los distintos grupos de edad con experiencias homosexuales y malestar asociado

\begin{tabular}{|c|c|c|c|c|c|}
\hline & & $\begin{array}{c}18-22 \\
(\%)\end{array}$ & $\begin{array}{c}23-27 \\
(\%)\end{array}$ & $\begin{array}{c}28-32 \\
(\%)\end{array}$ & $X^{2}(p)$ \\
\hline \multirow[t]{2}{*}{ Guapa } & & 100 & 100 & 100 & --- \\
\hline & Malestar & 30 & 20 & 16 & $1,67(0,434)$ \\
\hline \multirow[t]{2}{*}{ Atractiva } & & 100 & 100 & 96,7 & $2,02(0,364)$ \\
\hline & Malestar & 23,3 & 13,3 & 10,3 & $2,07(0,355)$ \\
\hline \multirow[t]{2}{*}{ Sueños } & & 93,3 & 100 & 96,7 & $2,06(0,355)$ \\
\hline & Malestar & 10 & 13,3 & 0 & $3,94(0,139)$ \\
\hline \multirow[t]{2}{*}{ Fantasías } & & 96,7 & 93,3 & 93,3 & $0,42(0,809)$ \\
\hline & Malestar & 17,2 & 7,1 & 0 & $5,67(0,059)$ \\
\hline \multirow[t]{2}{*}{ Deseo } & & 100 & 96,7 & 93,3 & $2,06(0,355)$ \\
\hline & Malestar & 13,3 & 6,9 & 3,6 & $1,94(0,379)$ \\
\hline \multicolumn{2}{|c|}{ Rel. sexuales } & 90 & 93,3 & 93,3 & $0,31(0,856)$ \\
\hline & Malestar & 7,4 & 3,6 & 3,6 & $0,58(0,747)$ \\
\hline
\end{tabular}

Por lo que respecta a las experiencias heterosexuales, pese a no observarse diferencias significativas entre los grupos de edad, el grupo de menos edad, así como el de mayor edad, revelan mayor malestar a nivel descriptivo. En general, el hecho de considerar guapa a una persona del sexo contrario fue la conducta más prevalente $y$, por el contrario, la menos frecuente fue el hecho de fantasear con alguien del otro sexo. Por otra parte, el hecho de tener relaciones sexuales heterosexuales fue la conducta que provocó un mayor malestar en los tres grupos de edad. En contraposición, las conductas que menos malestar mostraron en general fueron el hecho de haber considerado guapa o atractiva a una persona del otro sexo (véase la tabla 3). 
Tabla 3. Porcentaje de personas de los distintos grupos de edad con experiencias heterosexuales y malestar asociado

\begin{tabular}{lccccc}
\hline & & $\begin{array}{c}18-22 \\
(\%)\end{array}$ & $\begin{array}{c}23-27 \\
(\%)\end{array}$ & $\begin{array}{c}28-32 \\
(\%)\end{array}$ & $X^{2}(\mathrm{p})$ \\
\hline \multirow{2}{*}{ Guapa } & & $\mathbf{1 0 0}$ & $\mathbf{1 0 0}$ & $\mathbf{9 6 , 7}$ & $\mathbf{2 , 0 2}(\mathbf{0 , 3 6 4 )}$ \\
& Malestar & 10 & 6,7 & 3,4 & $1,01(0,604)$ \\
\hline \multirow{2}{*}{ Atractiva } & $\mathbf{8 0}$ & $\mathbf{9 6 , 7}$ & $\mathbf{8 6 , 7}$ & $\mathbf{3 , 9 3}(\mathbf{0 , 1 4 0 )}$ \\
& Malestar & 4,2 & 3,4 & 7,7 & $0,57(0,752)$ \\
\hline \multirow{2}{*}{ Sueños } & & $\mathbf{4 3 , 3}$ & $\mathbf{6 6 , 7}$ & $\mathbf{6 0}$ & $\mathbf{3 , 5 2}(\mathbf{0 , 1 7 1 )}$ \\
& Malestar & 23,1 & 15 & 11,1 & $0,82(0,661)$ \\
\hline \multirow{2}{*}{ Fantasías } & $\mathbf{2 0}$ & $\mathbf{4 6 , 7}$ & $\mathbf{3 3 , 3}$ & $\mathbf{4 , 8 0}(\mathbf{0 , 0 9 1})$ \\
& Malestar & 16,7 & 7,1 & 40 & $3,98(0,136)$ \\
\hline \multirow{2}{*}{ Deseo } & & $\mathbf{3 6 , 7}$ & $\mathbf{4 3 , 3}$ & $\mathbf{4 6 , 7}$ & $\mathbf{0 , 6 3 ( 0 , 7 2 7 )}$ \\
& Malestar & 18,2 & 0 & 7,1 & $2,72(0,256)$ \\
\hline \multicolumn{2}{l}{ Rel. sexuales } & $\mathbf{3 3 , 3}$ & $\mathbf{4 3 , 3}$ & $\mathbf{5 3 , 3}$ & $\mathbf{2 , 4 4}(\mathbf{0 , 2 9 5 )}$ \\
& Malestar & 40 & 15,4 & 50 & $3,83(0,147)$ \\
\hline
\end{tabular}

\section{Conclusiones}

A partir de los presentes resultados, queda patente la necesidad de flexibilizar las etiquetas sobre orientación sexual, ya que, como se ha observado en otros estudios (Copen, Chandra y Febo-Vazquez 2016; Nebot-Garcia, García-Barba, Gil-Juliá, Giménez-García y Ballester-Arnal 2018), podría no corresponder con la realidad. En concreto, nuestros hallazgos muestran cómo la población que se autoidentifica como homosexual tendría una sexualidad más diversa de lo que cabría esperar según las etiquetas normativas. De hecho, en nuestro estudio se observa que gran parte de la población homosexual ha considerado guapa o atractiva a una persona del otro sexo y más de un tercio ha mantenido relaciones sexuales heterosexuales.

La realización de estas conductas, así como de las conductas homosexuales, producen malestar a parte de la población homosexual. Una posible explicación puede ser la rigidez de las etiquetas sexuales (Epstein, McKinney, Fox y Garcia 2012; Thompson y Morgan 2008), que crean malestar en aquellas personas que realizan comportamientos que se salen de los esperados por su orientación sexual.

De forma más detallada, se ha observado que las puntuaciones más elevadas de malestar aparecen cuando existen relaciones sexuales heterosexuales. Podría ser debido a que estas conductas se realizan como parte del proceso de reafirmación de la propia orientación sexual, en un momento de grandes dudas, lo que podría generar el sentimiento de malestar (Soriano 2004).

En cuanto a las conductas homosexuales, se observa mayor porcentaje de malestar por considerar a alguien del mismo sexo como guapo o atractivo en comparación con el malestar experimentado por las relaciones sexuales homosexuales. Una posible explicación es que, cuando se llevan a cabo las relaciones sexuales, la persona ya ha pasado por un proceso madurativo y reflexivo acerca de sus intereses sexuales; en cambio, el hecho de considerar a alguien del mismo sexo como guapo o atractivo 
puede ser una experiencia más inconsciente e involuntaria, y haría plantearse dudas acerca de la propia orientación sexual.

No obstante, contrario a lo que se había observado en otros estudios (Kertzner, Meyer, Frost y Stirratt 2009; Luyckx, Klimstra, Duriez, Van Petegem y Beyers 2013), la edad no resultó una variable determinante en la realización de experiencias homosexuales y heterosexuales y el malestar asociado. Una posible causa podría ser que se tratara de una cultura diferente (Kertzner, Meyer, Frost y Stirratt 2009) o de que existiera una mayor diversidad en el nivel de estudios de los participantes (Luyckx, Klimstra, Duriez, Van Petegem y Beyers 2013). Del mismo modo, otra posible causa es que la edad no sea tan importante como la fase del proceso identitario en la que se encuentren (Soriano 2004). Pese a que se asume que cuanto mayor es la persona, más avanzado debe estar en el proceso, no tiene por qué ser así, por lo que se tendría que haber tenido en cuenta la etapa y no tanto la edad.

Por una parte, pese a la información que estos hallazgos proporcionan, habría que aumentar el número de participantes, ya que así se podrían obtener unos resultados más concluyentes y definitivos. Del mismo modo, también sería conveniente que los participantes estuvieran repartidos equitativamente en cuanto al nivel educativo, las creencias religiosas y la ideología política.

En cualquier caso, los resultados encontrados en este estudio hacen visible la necesidad de flexibilizar las categorías sexuales para promover una vivencia sana de la orientación sexual. Para ello, habría que investigar en mayor medida la formación de la identidad psicosexual, con el fin de elaborar programas de atención específicos para esta población que mitiguen el malestar experimentado.

\section{Referencias bibliográficas}

Bosson, Jennifer K., Jennifer L. Prewitt-Freilino y Jenel N. Taylor. 2005. «Role rigidity: A problem of identity misclassification?». Journal of personality and social psychology 89(4): 552-565.

Copen, Casey E., Anjani Chandra e Isaedmarie Febo-Vazquez. 2016. «Sexual behavior, sexual attraction, and sexual orientation among adults aged 18-44 in the United States: Data from the 2011-2013 National Survey of Family Growth». National Health Statistics Reports 88: 1-14.

Digoix, Marie, Marina Franchi, José Ignacio Pichardo Galán, Giulia Selmi, Maties de Stéfano Barbero, Matthias Thibeaud y Jose A. M. Vela. 2016. "Sexual orientation, family and kinship in France, Iceland, Italy and Spain». Families and Societies Working Paper 54(2016). Recuperado de: http://www.familiesandsocieties.eu/wpcontent/uploads/2016/08/WP54Digoixetal2016.pdf [Consultado el 13 de mayo de 2018].

Epstein, Robert, Paul McKinney, Shannon Fox y Carlos Garcia. 2012. «Support for a fluid-continuum model of sexual orientation: A large-scale Internet study». Journal of Homosexuality 59(10): 1356-1381.

Herz, Marcus y Thomas Johansson. 2015. "The normativity of the concept of heteronormativity». Journal of Homosexuality 62(8): 1009-1020.

Kertzner, Robert M., Ilan H. Meyer, David M. Frost y Michael J. Stirratt. 2009. «Social and Psychological Weil-Being in Lesbians, Gay Men, and Bisexuals: The Effects of Race, Gender, Age, and Sexual Identity». American Journal of Orthopsychiatry 79(4): 500-510. 
Luyckx, Koen, Theo A. Klimstra, Bart Duriez, Stijn Van Petegem y Wim Beyers. 2013. «Personal identity processes from adolescence through the late 20s: Age trends, functionality, and depressive symptoms». Social Development 22(4): 701-721.

Martxueta, Aitor y Juan Etxeberria. 2014. «Análisis diferencial retrospectivo de las variables de salud mental en lesbianas, gais y bisexuales (LGB) víctimas de bullying homofóbico en la escuela». Revista de Psicopatología y Psicología Clínica 19(1): 23-35.

Morgan, Elisabeth M., Matthew G. Steiner y Elizabeth Morgan Thompson. 2010. «Processes of sexual orientation questioning among heterosexual men». Men and Masculinities 12(4): 425-443.

Morgan, Elisabeth M. y Elizabeth Morgan Thompson. 2011. «Processes of sexual orientation questioning among heterosexual women». Journal of Sex Research 48(1): 16-28.

Nebot-Garcia, Juan Enrique, Marta García-Barba, Beatriz Gil-Juliá, Cristina GiménezGarcía y Rafael Ballester-Arnal. 2018. «Comportamientos homosexuales en jóvenes heterosexuales: diferencias de género». Revista Àgora de Salut 5: 5968.

Newcomb, Michael E. y Brian Mustanski. 2010. «Internalized homophobia and internalizing mental health problems: A meta-analytic review». Clinical Psychology Review 30(8): 1019-1029.

Soriano, Sonia. 2004. Cómo se vive la homosexualidad y el lesbianismo (2. ${ }^{a}$ edición). Salamanca: Amarú.

Thompson, Elizabeth Morgan y Elisabeth M. Morgan. 2008. «"Mostly straight" young women: Variations in sexual behavior and identity development». Developmental Psychology 44(1): 15-21. 